ARQGA / 927

\title{
A IMPORTÂNCIA DA
}

\section{ENDOSCOPIA DIGESTIVA ALTA} COM SOLUÇÃO DE LUGOL NO DIAGNÓSTICO DE CÂNCER SUPERFICIAL E DISPLASIA EM ESÔFAGO DE DOENTES COM NEOPLASIAS DE CABEÇA E PESCOÇO

\author{
Alfio José TINCANI*, Nelson BRANDALISE**, Nelson Adami ANDREOLLO**, \\ Luiz Roberto LOPES**, Ciro Garcia MONTES**, Albina ALTEMANI*** e Antonio S. MARTINS*
}

RESUMO - Os tumores localizados em região de cabeça e pescoço apresentam alta incidência em nosso meio, principalmente se associados com fatores etiológicos ligados aos hábitos de etilismo e tabagismo. Estes mesmos hábitos relacionam-se também com a incidência de câncer em esôfago. Assim sendo, é de se esperar que estes pacientes venham a apresentar outra neoplasia primária neste último órgão. Foi realizado estudo prospectivo com 60 pacientes portadores de câncer em mucosa da região de cabeça e pescoço na Disciplina de Cirurgia de Cabeça e Pescoço e no Serviço de Endoscopia Digestiva do Gastrocentro - UNICAMP, com o objetivo de diagnosticar neoplasias malignas superficiais no esôfago, caracterizadas como segundo tumor primário, e displasias. A endoscopia digestiva alta foi indicada utilizando-se a solução de lugol a 2\%, para coloração do epitélio esofagiano, evidenciando-se em 33 pacientes (55\%), 42 áreas iodoclaras (suspeitas de neoplasias), que foram submetidas a biopsias para estudo anatomopatológico. Foram diagnosticados cinco carcinomas superficiais de esôfago (8,3\%), sendo que três deles eram intra-epiteliais - in situ (5,0\%) e cinco áreas de displasia (8,3\%). Concluiu-se que o método de coloração da mucosa esofagiana com a solução de lugol a 2\%, através da endoscopia digestiva alta, apresenta alta sensibilidade e baixa especificidade para o diagnóstico de um segundo tumor primário e de displasia, em pacientes portadores de carcinoma em mucosa de região de cabeça e pescoço.

DESCRITORES - Neoplasias esofágicas. Endoscopia do sistema digestivo. Neoplasias de cabeça e pescoço. Coloração. Iodetos.

Trabalho realizado Disciplina de Cirurgia de Cabeça e Pescoço do Departamento de Cirurgia da Universidade Estadual de Campinas - UNICAMP, Campinas, SP.

* $\quad$ Serviço de Endoscopia Digestiva do Gastrocentro - UNICAMP.

** Departamento de Anatomia Patológica - UNICAMP.

*** Universidade Estadual de Campinas - UNICAMP, Campinas, SP.

Endereço para correspondência: Dr. Alfio José Tincani - Rua Prof. Achille Bassi, 140/6A, 13083-530 - Campinas, SP. e-mail: tincani@unicamp.br 
Tincani AJ, Brandalise N, Andreollo NA, Lopes LR, Montes CG, Altemani A, Martins AS. A importância da endoscopia digestiva com solução de lugol no diagnóstico de câncer superficial e displasia em esôfago de doentes com neoplasias de cabeça e pescoço

\section{INTRODUÇÃO}

Os tumores malignos da região de cabeça e pescoço são neoplasias que apresentam alta incidência na população brasileira, principalmente os localizados em cavidade oral ${ }^{(3,6,23)}$. Os fatores etiológicos desses tumores estão, na maioria dos casos, ligados aos hábitos de etilismo e tabagismo e seus portadores são tidos como grupos de risco para desenvolver uma segunda neoplasia tanto sincrônica, como metacrônica ao tumor primário. Entre as regiões alvos de um segundo tumor primário, as mais freqüentes são as localizadas no trato aerodigestivo alto, como o esôfago ${ }^{(14,15,16,21)}$.

Diante de doente portador de tumor primário em região de cabeça e pescoço, é de extrema importância o diagnóstico precoce de provável segundo tumor primário, visando-se melhor prognóstico e, portanto, o aumento da sua sobrevida.

A indicação rotineira da endoscopia digestiva alta e outros métodos com imagem tem tornado possível este diagnóstico. Porém, a acurácia desses exames melhora muito quando se usam artifícios técnicos, que propiciam maior chance para se diagnosticar precocemente estas prováveis lesões, principalmente em esôfago, onde áreas diminutas ou planas são difíceis de ser bem examinadas.

A solução de lugol a 2\%, instilada na luz esofágica com a finalidade de identificar áreas de mucosa suspeitas de neoplasias ou passíveis de transformação maligna, permitindo a realização de biopsias dirigidas, tem sido preconizada por vários autores ${ }^{(1,4,7,17,18,22,25)}$.

O uso da solução de lugol como corante para diagnóstico precoce de câncer foi primeiro utilizado por SCHILLER ${ }^{(13)}$, em 1933, no colo do útero. $\mathrm{O}$ autor relatou que o epitélio normal do colo uterino, cuja camada superficial contém glicogênio, pode ser corado com uma solução de lugol, adquirindo um aspecto escuro. Contudo, diante de processos patológicos o epitélio não se corou, permanecendo claro, o que foi denominado como área iodo-clara. A biopsia da área, na grande maioria das vezes, leva ao diagnóstico anatomopatológico da lesão.

O objetivo da presente pesquisa foi realizar estudo prospectivo, através da endoscopia digestiva alta com lugol, de doentes conhecidamente portadores de câncer em mucosa da região de cabeça e pescoço, com a finalidade de diagnosticar neoplasias malignas superficiais no esôfago, caracterizadas como segundo tumor primário, e displasias.

\section{CASUÍSTICA E MÉTODOS}

Durante o período desta pesquisa, iniciada em $1993^{(20)}, 60$ doentes em tratamento na Disciplina de Cirurgia de Cabeça e Pescoço do Departamento de Cirurgia da Universidade Estadual de Campinas UNICAMP e portadores de carcinoma epidermóide em mucosa da região da cabeça e pescoço foram selecionados para se submeterem a endoscopia digestiva, com coloração do epitélio do esôfago, utilizando- se a solução de lugol a 2\%, em período máximo estabelecido em 45 dias da data do diagnóstico do tumor primário. Todos os exames endoscópicos foram realizados no Gastrocentro da Faculdade de Ciências Médicas da UNICAMP. Nesta casuística, 55 (91,6\%) pacientes eram do sexo masculino, com faixa etária variando de 32 a 74 anos, média de 55,3 $\operatorname{anos}^{(20,21)}$.

Foram considerados os seguintes critérios de inclusão neste estudo: 1) doentes com diagnóstico comprovado por exame anatomopatológico, de carcinoma epidermóide em mucosa de região de cabeça e pescoço, sem tratamento anterior e em qualquer estádio; 2) etilistas (mais de três doses de aguardente ou equivalente ao dia) e tabagistas (mais de 10 cigarros ao dia), por período igual ou superior a 10 anos seguidos; 3) doentes assintomáticos quanto à doença localizada em esôfago.

A localização dos tumores primários na região de cabeça e pescoço está descrita na Tabela 1 .

TABELA 1 - Localização primária dos tumores em região de cabeça e pescoço

\begin{tabular}{lcr}
\hline Localização & Pacientes $(\mathbf{n})$ & $\mathbf{( \% )}$ \\
\hline Cavidade oral & 8 & $(13,3)$ \\
Orofaringe & 16 & $(26,6)$ \\
Hipofaringe & 10 & $(16,7)$ \\
Laringe & 26 & $(43,4)$ \\
Total & 60 & $(100)$ \\
\hline
\end{tabular}

No estadiamento dos tumores primários em cabeça e pescoço, foram utilizados os critérios da $\mathrm{UICC}^{(24)}$ de 1987, sendo que: 25 doentes pertenciam ao estádio IV; 23 ao estádio III, e 11 ao estádio II. Apenas um doente pertencia ao estádio I.

Os doentes selecionados para este estudo foram indagados por ocasião do exame clínico, quanto à sintomatologia esofagiana que pudesse estar correlacionada a provável câncer neste órgão. A seguir, foram submetidos a exame clínico minucioso, com ênfase a lesão em região de cabeça e pescoço. Identificado o tumor, foram indicadas biopsias para sua confirmação através do exame anatomopatológico. Além disso, foi rotina a realização de estudo radiográfico do tórax em duas incidências. A seguir, foi solicitado o exame endoscópico e coloração do esôfago com solução de lugol a $\%$.

Os doentes foram orientados a manter jejum mínimo de 6 horas seguidas e, imediatamente antes do exame, recebiam $3 \mathrm{~mL}$ de dimetilpolisiloxana por via oral e anestesia tópica com lidocaína "spray" a $10 \%$ em cavidade oral e orofaringe. Após o preparo e a sedação habituais, a endoscopia digestiva alta foi realizada com o videoendoscópio flexível da marca Olympus ${ }^{\circledR}$ (Tokyo, Japão). Inicialmente procedeu-se ao exame rotineiro do esôfago, estômago e duodeno e, a seguir, foi introduzido um cateter especial de polietileno, com "spray" tipo chuveiro em sua extremidade, através do canal de 
biopsia do endoscópio pelo qual instilaram-se $20 \mathrm{~mL}$ de água para a retirada de muco ou secreções existentes. O cateter foi, então, posicionado na região da cárdia e, sob visão direta do examinador, a solução de lugol foi homogeneamente distribuída em sentido retrógrado, até o terço superior do esôfago (Figura 1). O volume total da solução instilada não ultrapassou a $20 \mathrm{~mL}$. Após dois minutos de contato, o epitélio esofagiano foi novamente lavado com $40 \mathrm{~mL}$ de água, para a retirada de qualquer excesso da solução de lugol para melhor se evidenciarem as áreas iodo-claras e também fotografá-las (Figura 2). A mucosa esofágica normal cora-se em tom esverdeado ou marrom (a solução de lugol reage com o glicogênio presente no epitélio normal), enquanto as lesões malignas ou benignas não se coram e se apresentam claras ou amareladas, ou seja, padrão de coloração similar ao teste de SCHILLER ${ }^{(13)}$ usado para diagnosticar câncer do colo uterino.

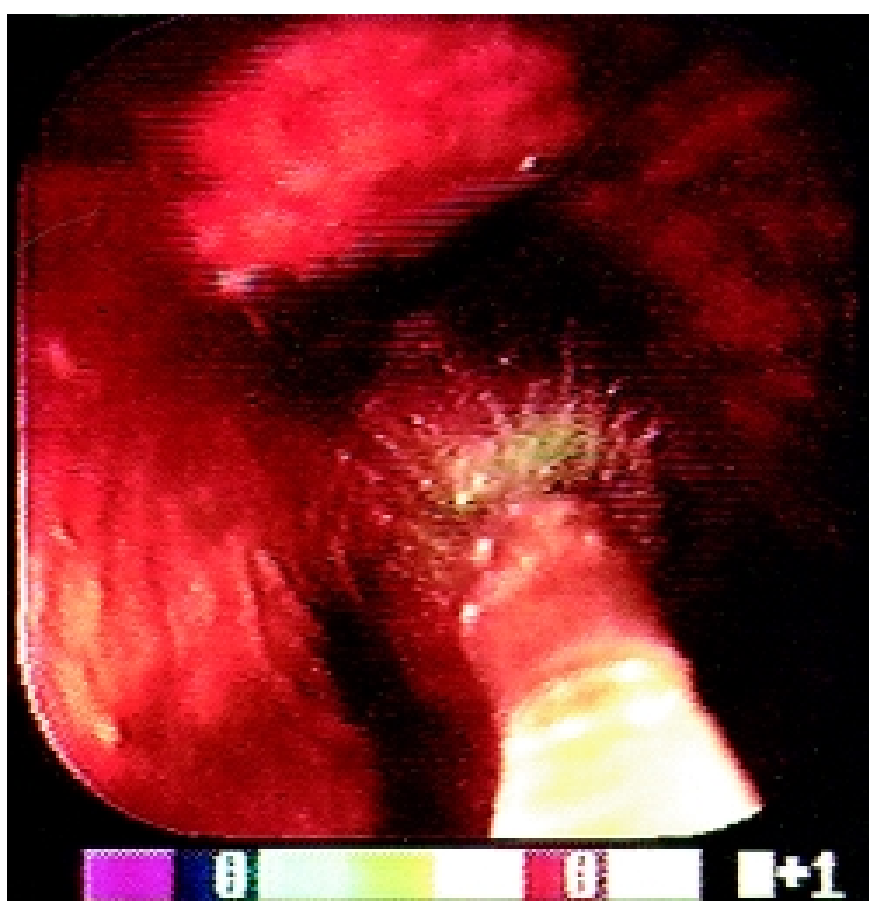

FIGURA 1 - Cateter de polietileno com ponta tipo chuveiro, instilando a solução de lugol a $2 \%$ no epitélio esofagiano. Notar área iodo-clara em lesão plana superiormente.

As áreas não-coradas ou coradas parcialmente (áreas suspeitas) foram submetidas a pelo menos, cinco biopsias, através de pinça do endoscópio, tipo saca-bocado. O material colhido foi fixado em formalina a $10 \%$ e enviado para exame anatomopatológico. Os fragmentos foram incluídos em parafina e as secções histológicas, coradas por hematoxilina-eosina.

Nos doentes com o diagnóstico de displasia, a coloração com o lugol e biopsias dirigidas foram indicadas e repetidas no mínimo a
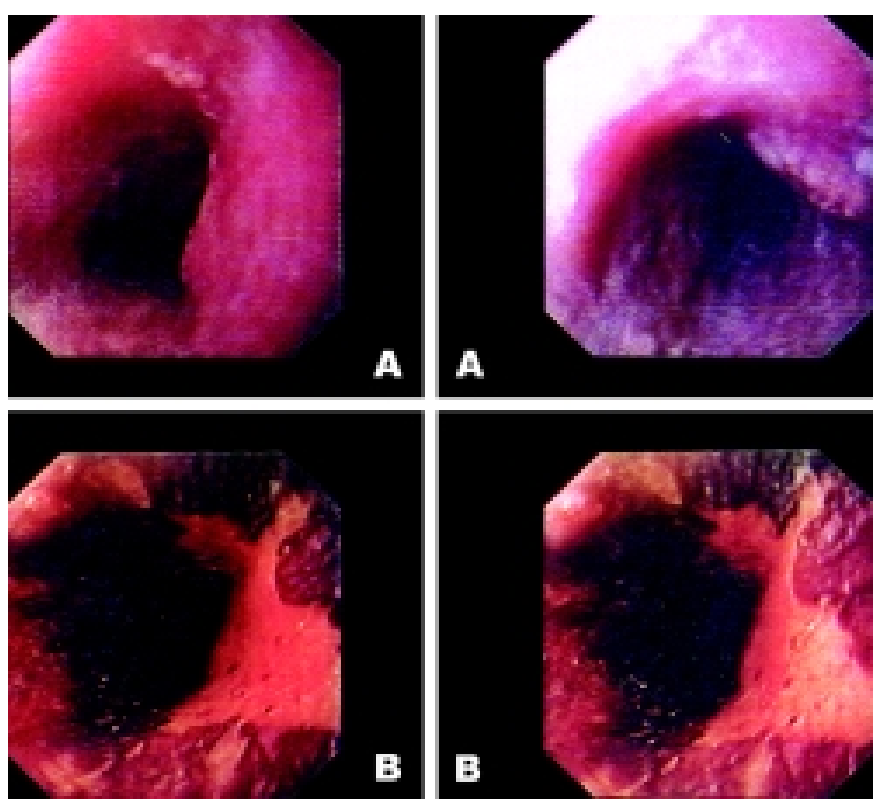

FIGURA 2 - Endoscopia digestiva alta, mostrando o epitélio esofágico com aparente aspecto normal (A). Em B, após a coloração com a solução de lugol a $2 \%$, nota-se área iodo-clara na mucosa do esôfago, contrastada com o epitélio normal em tom escuro.

cada 6 meses, enquanto para os pacientes considerados como epitélio esofágico normal ou com outras doenças benignas, o exame foi recomendado ao menos a cada 12 meses.

O conceito de displasia utilizado neste trabalho é o mesmo usado por ROBBINS ${ }^{(11)}$ e LEWIN et al..$^{(9)}$, onde as displasias são consideradas como lesões pré-cancerosas epiteliais. A displasia leve apresenta células atípicas, acometendo o terço basal da espessura do epitélio. O grau moderado caracteriza-se por apresentar células atípicas comprometendo os dois terços basais da espessura do epitélio. Na displasia grave, observa-se anaplasia das células que envolvem toda a espessura do epitélio, porém mantendo íntegra apenas uma fina camada superficial. A displasia grave é difícil de ser diferenciada do carcinoma intra-epitelial ou in situ.

\section{RESULTADOS}

Dentre os 60 doentes selecionados, $33(55,0 \%)$ apresentaram áreas iodo-claras (suspeitas de neoplasia) no esôfago. Nestes casos, foram observadas 42 destas áreas, sendo que sete doentes apresentaram duas e um deles, três áreas iodo-claras. A maioria das áreas evidenciadas pela coloração com a solução de lugol, $28(66,6 \%)$ estavam localizadas em esôfago médio, nove (21,4\%), em esôfago inferior e cinco (12,0\%), em esôfago superior. 
Tincani AJ, Brandalise N, Andreollo NA, Lopes LR, Montes CG, Altemani A, Martins AS. A importância da endoscopia digestiva com solução de lugol no diagnóstico de câncer superficial e displasia em esôfago de doentes com neoplasias de cabeça e pescoço

O carcinoma superficial foi definido como um carcinoma esofágico restrito a camada submucosa e, carcinoma intra-epitelial, como uma lesão restrita a camada epitelial (in situ). Assim, foram encontrados cinco doentes $(8,3 \%)$ com carcinoma do esôfago. Em três, a lesão era intra-epitelial (5,0\%) (Figura 3) e nos demais (3,3\%) já invadia até a camada muscular da mucosa do esôfago (Figura 4). Todas estas áreas estavam localizadas em esôfago médio e, portanto, os doentes foram submetidos a esofagectomia com esofagogastroplastia da região cervical, após o tratamento cirúrgico do câncer primário de cabeça e pescoço. Em um doente foi realizada cirurgia simultânea para os dois tumores (faringolaringoesofagectomia total), reconstituindo-se o trânsito digestivo com a elevação do estômago na orofaringe (anastomose faringogástrica). $\mathrm{O}$ exame histopatológico das peças cirúrgicas ressecadas confirmou tratar-se de carcinoma epidermóide. Em quatro doentes, o câncer de esôfago foi considerado sincrônico e em um foi metacrônico ao tumor da região de cabeça e pescoço.

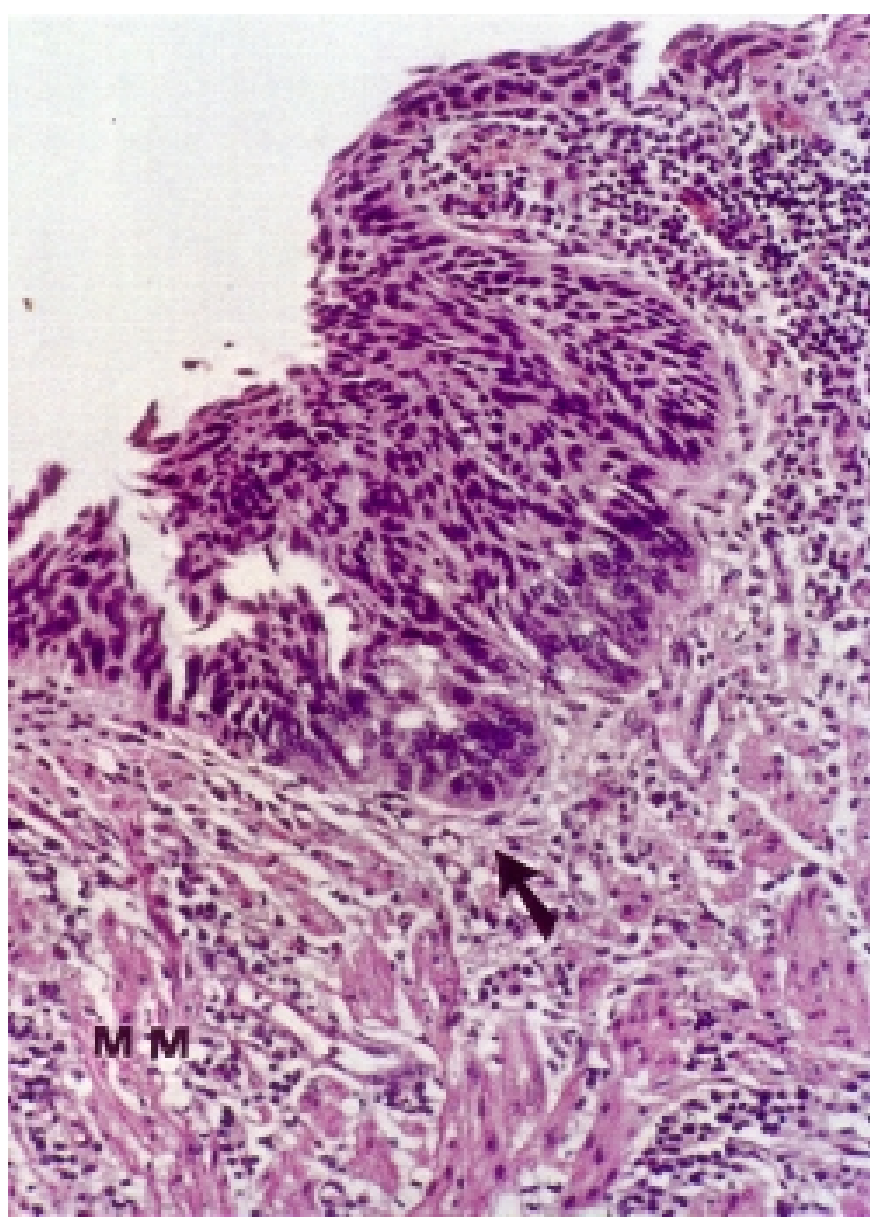

FIGURA 3 - Microfotografia, mostrando carcinoma superficial intraepitelial do esôfago. Seta = limite da lesão intra-epitelial. $\mathrm{MM}=$ muscular da mucosa (HE x330).

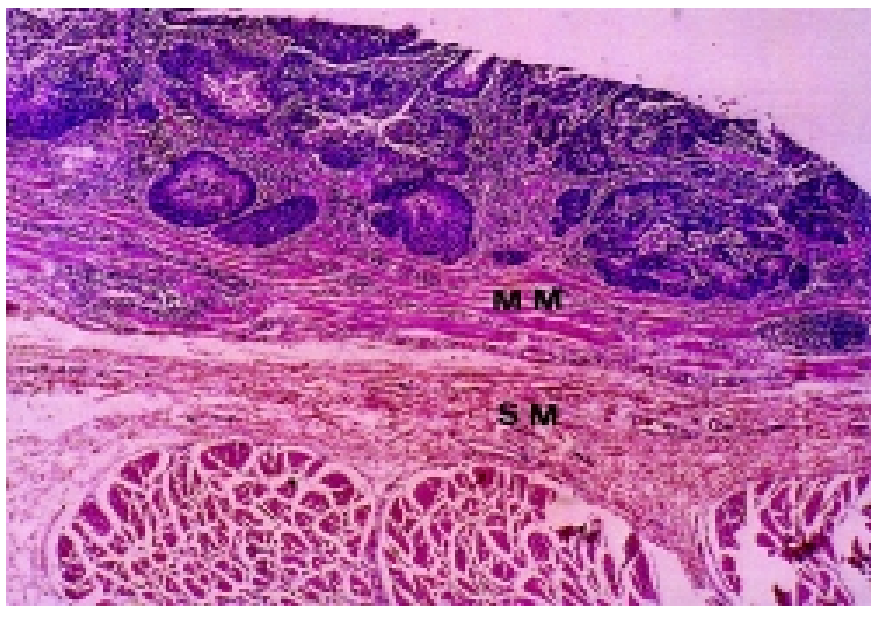

FIGURA 4 - Microfotografia, mostrando carcinoma superficial com invasão neoplásica até a camada muscular da mucosa do esôfago. $\mathrm{MM}=$ muscular da mucosa e $\mathrm{SM}=$ submucosa (HE x85).

Além disso, cinco outros doentes $(8,3 \%)$ apresentaram displasia do epitélio esofagiano. Três foram classificadas como displasias leves e dois, como moderadas.

As outras 32 áreas não-coradas foram classificadas como esofagites em 22, epitélio de Barrett em três e lesões inespecíficas nos sete doentes remanescentes.

\section{DISCUSSÃO}

Os doentes com tumores da região de cabeça e pescoço têm risco elevado para a ocorrência de uma segunda neoplasia primária, localizada principalmente em pulmões e esôfago. Quando esta ocorrência é no esôfago e as lesões são superficiais, as técnicas habituais de realização do exame radiográfico contrastado tornam difícil o diagnóstico, pois podem ser planas ou diminutas, proporcionando poucas alterações radiológicas ${ }^{(1,14)}$. O mesmo pode ocorrer durante a endoscopia digestiva, pois as lesões com este aspecto e sem aparente perda de continuidade da mucosa, podem passar despercebidas $^{(8,10)}$.

LEWIN et al. ${ }^{(9)}$ descreveram que, pela endoscopia, os vários tipos de tumores do esôfago, em $60 \%$ dos casos, têm aspecto exofítico. O tumor infiltrativo é menos comum e representa cerca de 15,0\%. Quando o carcinoma é superficial, com invasão limitada à lâmina própria ou até à submucosa, pode aparecer uma lesão protrusa, erosiva ou ulcerada, com aspecto granuloso e, por vezes, plano, tornando-se invisível à endoscopia digestiva.

O emprego da endoscopia digestiva associado à solução de lugol, para corar o esôfago dos pacientes com câncer de cabeça e pescoço, foi proposto por SHIOZAKI et al. ${ }^{(17)}$, INA et al. ${ }^{(8)}$ e YOSHIDA et al. ${ }^{(25)}$. 
Tincani AJ, Brandalise N, Andreollo NA, Lopes LR, Montes CG, Altemani A, Martins AS. A importância da endoscopia digestiva com solução de lugol no diagnóstico de câncer superficial e displasia em esôfago de doentes com neoplasias de cabeça e pescoço

SHIOZAKI et al. ${ }^{(17)}$ descreveram o estudo de 178 pacientes portadores de neoplasias malignas de cabeça e pescoço, tratados da doença inicial e que realizaram endoscopia digestiva alta, com o uso da solução de lugol. Foram diagnosticados nove carcinomas superficiais $(5,1 \%)$, sendo quatro sincrônicos $(2,3 \%)$ e cinco metacrônicos $(2,8 \%)$.

INA et al. ${ }^{(8)}$ utilizaram a solução de lugol a $2 \%$ para o estudo do esôfago de 127 pacientes, todos do sexo masculino e portadores de neoplasia maligna em cabeça e pescoço. Destes, 101 apresentavam câncer de cavidade oral e 26 , de orofaringe. Oito deles $(6,3 \%)$ apresentaram carcinoma superficial de esôfago concomitante, pela sua descrição, com os tumores de cabeça e pescoço. Segundo os mesmos autores, os pacientes do sexo masculino com carcinoma epidermóide de cabeça e pescoço situado em cavidade oral e orofaringe, têm 10 vezes mais risco do que os do sexo feminino de apresentar câncer sincrônico ou metacrônico em esôfago.

TONETO Jr ${ }^{(22)}$, em estudo também bem conduzido no Rio Grande do Sul, onde a incidência de carcinoma do esôfago é elevada, empregando metodologia semelhante de cromoendoscopia e analisando 43 doentes com tumores de cabeça e pescoço, encontrou sete carcinomas epidermóides (16,3\%) e uma área de displasia $(2,3 \%)$.

Quanto ao diagnóstico de lesões displásicas no esôfago é de extrema importância a utilização do método, pelo risco destas virem a transformar-se em carcinomas ${ }^{(8,9,11,12,19)}$ e também porque são áreas planas, de difícil avaliação através de estudo radiográfico com bário e de endoscopia ${ }^{(8,10,14,23)}$. ANTONIOLI ${ }^{(2)}$ descreveu que, em doentes com displasia grave em esôfago, $30 \%$ destas progridem para carcinoma invasivo. Na displasia leve, esta progressão é de $15 \%$. O mesmo autor relatou que em portadores de esofagite crônica e displasia, 34\% destas podem evoluir para carcinoma. Quando há somente a existência de esofagite crônica, a progressão para câncer é de 4\%.

Neste estudo, dos cinco pacientes com diagnóstico endoscópico de carcinoma superficial de esôfago ao teste com lugol, apenas um teve a lesão suspeita vista pela endoscopia convencional. Foram diagnosticadas cinco displasias no esôfago, sendo que, em um deles, após 14 meses do diagnóstico de displasia moderada, em novo exame com endoscopia e uso da solução de lugol seguido de biopsias, revelou carcinoma epidermóide intra-epitelial. Esta observação confirma relatos de outros autores ${ }^{(12)}$ de que a transformação neoplásica pode ocorrer. Similarmente, em outro paciente, 25 meses após o uso do método, obteve-se o diagnóstico de displasia leve em área de esofagite ${ }^{(21)}$. Nos demais pacientes não houve alteração anatomopatológica durante o seguimento realizado.

Assim, baseado nestas observações recomenda-se que todos os pacientes com áreas não-coradas, porém com diagnóstico de lesão benigna e com áreas coradas (normais) no esôfago tenham uma nova endoscopia com lugol no mínimo a cada 12 meses. Para doentes com displasia, a endoscopia, uso do corante lugol e biopsias devem ser repetidas em intervalos não superiores a seis meses.
O câncer de esôfago por ser uma neoplasia muito agressiva, com características de invasão precoce na submucosa e ocorrência de metástases, tanto linfáticas como hematogênicas, tem sobrevida pequena (10 a $20 \%$ em 5 anos), a despeito do tratamento instituído ${ }^{(17)}$. Porém, ao tratar-se doentes com diagnóstico precoce de tumores, a sobrevida, em 5 anos, pode chegar a 100\%, segundo estudos de ENDO et $\mathrm{al}^{(4)}$, e a $80 / 90 \%$, de acordo com SUGIMACHI et al. ${ }^{(18)}$. Portanto, o diagnóstico precoce nos doentes assintomáticos e com fatores de risco evidentes, pode elevar sua sobrevida.

SHIOZAKI et al. ${ }^{(17)}$ trataram com esofagectomia oito dos nove pacientes com câncer de esôfago superficial, associado ao de cabeça e pescoço. Dois pacientes apresentavam tumor sincrônico com câncer de laringe, sendo realizadas, assim, laringectomia e esofagectomia, simultaneamente. Um paciente, com tumor de glândula salivar, realizou radioterapia e esofagectomia. $\mathrm{O}$ mesmo tratamento foi feito com outro doente, com câncer de cavidade oral (radioterapia, no tumor primário, e esofagectomia). Dos cinco pacientes com tumores metacrônicos, quatro foram esofagectomizados, sendo que um teve seu tumor de esôfago ressecado por endoscopia digestiva e por laser, devido a problemas cardiovasculares graves. Os mesmos autores consideram que maior sobrevida ocorre após o tratamento cirúrgico de um tumor precoce de esôfago, associado a tratamento, com sucesso, da neoplasia de cabeça e pescoço.

INA et al. ${ }^{(8)}$ diagnosticaram oito casos de câncer superficial no esôfago, em 127 pacientes com neoplasia de cabeça e pescoço. Dois deles realizaram esofagectomia e na época da publicação do referido trabalho, estavam livres da doença. Os seis pacientes restantes foram tratados com quimioterapia e radioterapia. Destes, três estavam vivos, dois sem e um com a doença. Os outros três morreram por carcinoma em língua, orofaringe e reto, respectivamente, mas não pelo câncer de esôfago. Dois destes pacientes foram para necropsia, sendo que, em um, havia carcinoma superficial no esôfago, mesmo após a quimioterapia e a radioterapia.

Quando a neoplasia não está localizada em laringe ou hipofaringe, preconiza-se o tratamento do tumor primário da região de cabeça e pescoço e, posteriormente o da neoplasia superficial do esôfago ${ }^{(8,17)}$. Por serem cirurgias extensas, com morbidade alta, diminuem-se, assim, as chances de complicações no pós-operatório. $\mathrm{Na}$ presença de tumores localizados em laringe ou hipofaringe, pela continuidade destes com o esôfago, a cirurgia em monobloco é justificada. Em um dos pacientes com carcinoma intra-epitelial, realizou-se a ressecção endoscópica utilizando bisturi elétrico com sucesso. Não realizamos este procedimento rotineiramente, devido o carcinoma de esôfago ser multicêntrico ${ }^{(17)}$ e também por não contarmos no momento com endoultra-som, para certificarmos da verdadeira espessura da lesão.

A endoscopia digestiva alta é um método muito difundido no Brasil. Centros de pequeno e médio porte possuem médicos 
especialistas com capacidade para realizar o exame e diagnosticar as mais variadas doenças do trato digestivo alto.

A solução de lugol a $2 \%$ empregada é muito fácil de ser obtida e de baixo custo, pois é a mesma utilizada no teste de SCHILLER ${ }^{(13)}$ para o diagnóstico precoce do câncer de colo uterino.

Outros estudos realizados no Rio Grande do Sul por FAGUNDES $^{(5)}$ e FREITAG ${ }^{(7)}$, em populações de risco elevado de câncer de esôfago, comprovam a eficácia do emprego da solução de lugol associada à endoscopia no diagnóstico de lesões pré-cancerosas e neoplasias precoces.

Em doentes portadores de câncer primário da mucosa da região da cabeça e pescoço, a endoscopia digestiva alta associada ao lugol deve ser indicada de rotina tanto para identificar precocemente o câncer superficial e/ou displasia no esôfago, como para seguimento tardio dos mesmos. É um método praticamente livre de complicações. Em pacientes com esofagites severas ou lesões erosivas, pode ocorrer sensação de queimação em região abdominal alta ou tórax, sendo estes efeitos minimizados com a administração de água ou ainda protetores gástricos. Não é um método específico para diagnóstico de câncer precoce de esôfago, mesmo porque outras doenças podem ocasionar áreas nãocoradas. Contudo, o método tem alta sensibilidade quando câncer ou displasia estão presentes no epitélio do esôfago, pois não se têm resultados falso-negativos.

\section{AGRADECIMENTOS}

Os autores agradecem às acadêmicas Ana Paula Damiano e Rebecca C. K. Maunsell e aos residentes da Disciplina de Cirurgia de Cabeça e Pescoço.

Tincani AJ, Brandalise N, Andreollo NA, Lopes LR, Montes CG, Altemani A, Martins AS. The importance of the upper digestive endoscopy using lugol dye solution for diagnosis of the superficial esophageal cancer and dysplasia in patients with head and neck cancer. Arq Gastroenterol 2000;37(2):107-113.

ABSTRACT - Head and neck cancer has a high incidence in Brazil, with cancer of the oral cavity being one of the five most common cancers among Brazilians. Alcohol and tobacco consumption may contribute to synchronous or metachronous head and neck cancer and esophageal cancer. A prospective study involving 60 patients with head and neck cancer was carried out at the State University of Campinas UNICAMP, Campinas, SP, Brazil to screen for superficial esophageal cancer and dysplasia using endoscopy and a $2 \%$ lugol dye solution followed by biopsy of the suspicious areas. Five patients (8.3\%) had superficial esophageal cancer, which was diagnosed as intraepithelial carcinoma in three of them (5.0\%). In four patients, the superficial esophageal cancer was synchronous and in one it was metachronous to head and neck cancer. Five patients (8.3\%) had dysplasias in the esophageal epithelium (three were classified as mild and two as moderate). These results demonstrate the value of endoscopic screening of the esophagus using lugol dye in patients with head and neck cancer, particularly since superficial esophageal cancer is extremely difficult to detect by conventional methods in asymptomatic patients.

HEADINGS - Esophageal neoplasms. Endoscopy, digestive system. Head and neck neoplasms. Dyes. Iodates. 


\section{REFERÊNCIAS BIBLIOGRÁFICAS}

1. Adachi Y, Kitamura K, Tsutsui S, Ikeda H, Matsuda H, Sugimachi K. How to detect early carcinoma of the esophagus. Hepatogastroenterology, 1993;40:207.

2. Antonioli DA. Esophagus. In: Henson DE, Albores-Saavedra J, editors Pathology of incipient neoplasia. 2.ed. New York: W. B. Saunders; 1993. p.64-71.

3. Brumini R, Torloni H, Gotlieb SLD, Henson DE, Souza JMP. Câncer no Brasil: dados histopatológicos 1976-80. Rio de Janeiro, Campanha Nacional de Combate ao Câncer. Ministério da Saúde, 1982.

4. Endo M, Takeshita K, Yoshida M. How can we diagnose early stage of esophageal cancer? Endoscopy 1986;18 Suppl. 3:11.

5. Fagundes RB. Identificação de displasias em indivíduos sob risco para o carcinoma epidermóide de esôfago através da cromoendoscopia com lugol [dissertação]. Porto Alegre: Universidade Federal do Rio Grande do Sul; 1996.

6. Franco EL, Kowalsky LP, Oliveira BV, Curado MP, Raimunda NP, Silva ME, Fava AS, Torloni H. Risk factors for oral cancer in Brazil: a case control study. Int J Cancer 1989;43:992.

7. Freitag CPF. Cromoendoscopia com lugol no diagnóstico de displasias ocultas no esôfago: estudo em indivíduos sob risco para o carcinoma epidermóide esofágico portadores de alterações citológicas detectadas pelo balão chinês [dissertação]. Porto Alegre (RS): Universidade Federal do Rio Grande do Sul; 1997.

8. Ina H, Shibuya H, Ohashi I, Kitagawa M. The frequency of a concomitant early esophageal cancer in male patients with oral and oropharyngeal cancer. Cancer 1994; 73:2038.

9. Lewin KJ, Riddell RH, Weinstein WM. Gastrointestinal pathology and its clinical implications. Tokyo: Igaku-Shoin; 1992. p.440-92.

10. Misumi A, Harada K, Murakami A, Arima K, Kondo H, Akagi M, Yagi Y, Ikeda T, Kobori Y, Matsukane H, Baba K. Early diagnosis of esophageal cancer. Ann Surg 1989;210:732.

11. Robbins SL. Neoplasias. In: Cotran RS, Kumar V, Robbins SL, Schoen FJ. Pathologic basis of disease. 5.ed. New York: W. B. Saunders; 1994. p.247.

12. Rubio CA, Liu F, Zhao HZ. Histological classification of intraepitelial neoplasias and microinvasive squamous carcinoma of the esophagus. Am J Surg Pathol 1989;13:685.

13. Schiller W. Early diagnosis of carcinoma of the cervix. Surg Gynecol Obstet 1933;56:210.

14. Schmidt LW, Patrick JD, Wilson RT. Superficially invasive squamous cell carcinoma of the esophagus: a study of seven cases in Memphis, Tennessee. Gastroenterology 1986;91:1456.
15. Schottenfeld D. Alcohol as a co-factor in the etiology of cancer. Cancer 1979;43:1962.

16. Shaha AR, Hoover EL, Mitrani M, Marti JR, Krespi YP. Syncronicity, multicentrycity, and metachronicity of head and neck cancer. Head \& Neck Surg 1988;10:225.

17. Shiozaki H, Tahara H, Kobayashi K, Yano H, Tamura S, Imamoto H, Yano Y, Oku K, Miyata M, Nishiyama K, Kubo K, Mori T. Endoscopic screening of early esophageal cancer with the lugol dye method in patients with head and neck cancers. Cancer 1990;66:2068.

18. Sugimachi K, Ohno S, Matsuda H, Mori M, Kuwano H. Lugol-combined endoscopic detection of minute malignant lesions of the thoracic esophagus. Ann Surg 1988;208:179-83.

19. Takiyama W, Moriwari K, Mandai K, Takashima S. Relationship of esophagea dysplasia to associated head and neck cancer with esophageal carcinoma. Jpn J Clin Oncol 1996;26:12.

20. Tincani AJ, Martins AS, Steck JH, Franco EM, Brandalise NA. Protocolo para diagnóstico precoce de segundo tumor primário em esôfago, nos indivíduos portadores de neoplasia em cabeça e pescoço. In: Anais do $15^{\circ}$ Congresso Brasileiro de Cirurgia de Cabeça e Pescoço; 1993; Natal, RN. Natal: RN; 1993. p.222.

21. Tincani AJ. Diagnóstico do câncer superficial e de lesões displásicas de esôfago sincrônicas com o câncer da região mucosa de cabeça e pescoço com o emprego de exame endoscópico e coloração com solução de lugol a 2\% [Tese de Doutorado]. Campinas (SP): Universidade Estadual de Campinas; 1997.

22. Toneto Jr JE. Carcinoma epidermóide de esôfago associado às neoplasias de cabeça e pescoço. Estudo da freqüência e valor da cromoendoscopia no diagnóstico de lesões precursoras e neoplásicas assintomáticas [Dissertação]. Porto Alegre (RS): Universidade Federal do Rio Grande do Sul; 1998.

23. Torloni H, Brumini R. Freqüência e distribuição dos tumores malignos primários: generalidades em cabeça e pescoço. In: Registro nacional de tumores. Rio de Janeiro: Divisão Nacional de Doenças Crônico-Degenerativas. Ministério da Saúde; 1978

24. UICC - International Union Against Cancer. In: Hermanek P, Sobin LH, editors TNM classification of malignant tumors. 4.ed. Berlin: Springer-Verlag; 1987.

25. Yoshida M, Hanashi T, Hirose T, Inada K. Endoscopic iodine staining test for early detection of esophageal cancer for patients with malignants lesions and after treatment. Stomach \& Intestine (Tokyo) 1994;29:905. 